\title{
Article
}

\section{Superficial Venous Reflux Intervention Guided by Triggered Angiography Non-Contrast-Enhanced Sequence Magnetic Resonance Imaging: Different QFlow Pattern from Health Controls}

\author{
Chien-Wei Chen ${ }^{1}$, Yuan-Hsi Tseng ${ }^{2}$, Yueh-Fu Fang ${ }^{3}{ }^{\mathbb{D}}$, Min Yi Wong ${ }^{2}$, Yu-Hui Lin ${ }^{2}$ and Yao-Kuang Huang ${ }^{2, *},+\mathbb{C}$ \\ 1 Department of Diagnostic Radiology, Chia-Yi Gung Memorial Hospital and Chang Gung University, \\ College of Medicine, Taoyuan 333, Taiwan; chienwei33@gmail.com \\ 2 Division of Thoracic and Cardiovascular Surgery, Chia-Yi Chan Gung Memorial Hospital and \\ Chang Gung University, College of Medicine, Taoyuan 333, Taiwan; 8802003@cgmh.org.tw (Y.-H.T.); \\ mynyy001@gmail.com (M.Y.W.); vw200162@gmail.com (Y.-H.L.) \\ 3 Department of Thoracic Medicine, Linkou Chang Gung Memorial Hospital and Chang Gung University, \\ College of Medicine, Taoyuan 333, Taiwan; dr.fang.yf@gmail.com \\ check for \\ updates \\ Citation: Chen, C.-W.; Tseng, Y.-H.; \\ * Correspondence: huang137@icloud.com \\ + Current address: Department of Cardiovascular Surgery, Chia-Yi Chang Gung Memorial Hospital, \\ West Section, Chia-Pu Rd., Putzu City 61363, Chiayi, Taiwan.
} Fang, Y.-F.; Wong, M.Y.; Lin, Y.-H.; Huang, Y.-K. Superficial Venous Reflux Intervention Guided by Triggered Angiography Non-Contrast-Enhanced Sequence Magnetic Resonance Imaging: Different QFlow Pattern from Health Controls. J. Pers. Med. 2021, 11, 751. https://doi.org/10.3390/jpm11080751

Academic Editors:

Miguel Angel Ortega, Julia Bujan,

Melchor Alvarez-Mon,

Miguel A Alvarez-Mon,

Jorge Monserrat and

Natalio García-Honduvilla

Received: 2 July 2021

Accepted: 29 July 2021

Published: 30 July 2021

Publisher's Note: MDPI stays neutral with regard to jurisdictional claims in published maps and institutional affiliations.

Copyright: (C) 2021 by the authors. Licensee MDPI, Basel, Switzerland. This article is an open access article distributed under the terms and conditions of the Creative Commons Attribution (CC BY) license (https:/ / creativecommons.org/licenses/by/ $4.0 /)$.
Abstract: (1) Background: To assess the effectiveness of triggered angiography non-contrast-enhanced (TRANCE)-magnetic resonance imaging (MRI) in superficial venous reflux and its difference from health controls. (2) Methods: Thirty patients underwent TRANCE MRI before surgical intervention of their superficial venous reflux of the legs. Ten healthy volunteers were included as a control. (3) Results: TRANCE MRI involves the major tributaries, thus enhances the additional ablations in $20 \%$ of patients. QFlow pattern of superficial venous reflux (QFlow GSV / PV MF ratio > 1) was compared with the duplex scan (SFJ reflux) using Cohen's kappa coefficient at 0.967 . The 30 morbid legs undergoing TRANCE MRI-guide interventions and the healthy volunteers' legs on the same side were compared. The stroke volumes (SV) are higher in EIV $(p=0.021)$ in the left-leg-intervention group. The mean flux (MF) is higher in the EIV $(p=0.012)$ and trend of increasing in GSV segment $(p=0.087)$ in the left-leg-intervention group. The QFlow of 10 patients with right leg intervention are higher in GSV in the right-leg-intervention group (SV $p=0.002$; FFV $p=0.001$; MF $p=0.001$ ). QFlow data is shown for all legs for superficial venous intervention with GSV/PV (MF) ratio $>1$. (4) Conclusions: Typical figures in QFlow (GSV/PV MF ratio > 1) could be observed in the morbid limbs but not in the controls.

Keywords: MRI; personalized; endovascular; QFlow; varicose; reflux

\section{Introduction}

Venous diseases of the lower extremities include minor varicose veins and static ulcers, ranging from ambulatory venous hypertension, vascular compression (May Thurner syndrome) to potentially fatal status (such as deep vein thrombosis plus pulmonary emboli) [1-6]. Only a few modalities are available for objective venous evaluation of the lower limbs. The venous system is not precisely enhanced on the computed tomography (CT) venogram, and high-quality enhancement requires specific access (from a morbid limb). Compared with conventional angiography, most magnetic resonance venography (MRV) techniques involving contrast media have exhibited higher sensitivity in detecting lesions in vessels [7]. The triggered angiography non-contrast-enhanced (TRANCE) technique records differences in the vascular signal intensity during the cardiac cycle for subsequent image subtraction and provides a vascular image without requiring contrast agents. The clinical application of this technique has enabled the evaluation of the anatomical structure 
of the whole venous system in the lower extremities [6,8,9]. TRANCE-MRI reveals the location of not only venous compression but also all major collateral veins, thus helping to achieve superior venous ablation results. The interventions of the superficial venous reflux could be well discussed and be personalized according to the patients' preference. We integrated this technique into surgical planning for superficial venous reflux of the legs and summarized the value of this protocol (Supplementary Video S1).

\section{Materials and Methods}

\subsection{Patients}

The Institutional Review Board (IRB) of Chang Gung Memorial Hospital approved this study (IRB number: 201802137B0, 202001213B0 and 202100938B0), which included consecutive patients who received TRANCE-MRI for the evaluation of the venous diseases of their lower extremities at a tertiary hospital between April 2017 and May 2021. We prospectively collected and retrospectively analyzed their data to determine their clinical significance. All patients were suspected to have venous problems in their lower extremities. Patients were excluded if they exhibited poor compliance or had multiple comorbidities that prevented them from lying down for the whole TRANCE-MRI exam. Initially, 230 patients underwent TRANCE-MRI for the venous examination of their leg. Segmental QFlow hemodynamic and morphological examinations were performed in 30 patients before their superficial venous intervention.

Noninvasive color Doppler ultrasonography (US) and TRANCE-MRI were performed in all 30 patients to assess the venous status of their lower extremities before the scheduled superficial venous intervention (Figure 1). Doppler US was performed in the supine position. The femoral vein (FV), great saphenous vein (GSV), popliteal vein (PV), and perforating vein in the calves were examined. Pelvic veins were not evaluated in the Doppler examination. For further QFlow comparisons, we included 10 healthy volunteers in this study.

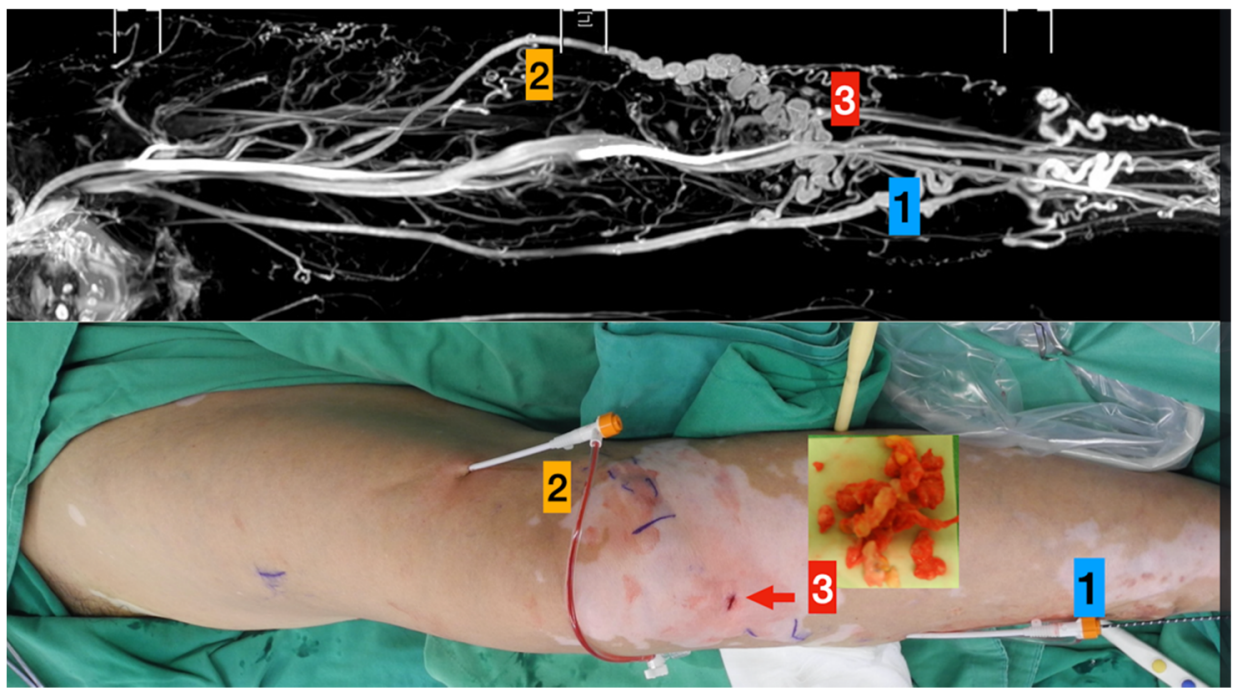

Figure 1. TRANCE MRI-Guiding superficial venous intervention.

Upper column (TRANCE MRI venous map):

1. Left great saphenous vein

2. Accessory saphenous vein

3. Major communicating tributaries.

Lower column (Surgical photos)

1. Primary truncal ablation of the great saphenous vein.

2. Additional ablation of the accessory saphenous vein.

3. Phlebectomy though the small incisions (red arrow). 


\subsection{MRI Acquisition}

MRI was performed using a 1.5-T MRI scanner (Philips Ingenia, Philips Healthcare, Best, the Netherlands). The process was carried out with the patients in supine; a peripheral pulse unit trigger was used. The arterial system images were evaluated through a three-dimensional (3D) turbo spin-echo (TSE) skill during systole and diastole periods. TSE TRANCE imaging was conducted using the following parameters: repetition time (TR), 1 beat; echo time (TE), shortest; flip angle, $90^{\circ}$; voxel size, $1.7 \mathrm{~mm} \times 1.7 \mathrm{~mm} \times 3 \mathrm{~mm}$; and field of view $(\mathrm{FOV}), 350 \times 420$. The relatively fast arterial blood flow during systole can cause signal dephasing and lead to flow voids. Accordingly, when systolic triggering is applied, the arteries appear black. The relatively slow arterial blood flow during diastole does not cause signal dephasing. Hence, the arteries appear bright on diastolic scans. Subtracting the two phased scans yields a 3D data set of the arteries only. Other images of the venous systems are evaluated through 3D TSE short-tau inversion recovery (STIR) during the systole period. TSE STIR TRANCE imaging was performed using the following parameters: TR, 1 beat; TE, 85; inversion recovery delay time, 160; voxel size, $1.7 \mathrm{~mm} \times 1.7 \mathrm{~mm} \times 4 \mathrm{~mm}$; and FOV, $360 \times 320$. STIR gives additional background suppression by suppressing connective tissues. When systolic triggering is applied, the arteries appear black. The imaging process yields a 3D data set of the venous system, and no subtraction is required for the data set. A quantitative flow scan is routinely performed to determine appropriate trigger delay times for systolic and diastolic triggering. All images were acquired without the use of gadolinium contrast medium. A QFlow scan entails several acquisitions occurring within one cardiac cycle, resulting in multiple phases. QFlow analysis provides information regarding stroke volume (SV), forward and backward flow volumes, flux, stroke distance (SD), mean velocity (MV), and vessel area. In this study, the postprocessing package calculated quantitative information such as flow velocity, visualized as two-dimensional flow maps overlaid on anatomical references. The bilateral external iliac veins (EIVs), FVs, PVs, and GSVs were analyzed.

\subsection{Statistical Analysis}

Continuous variables (age and QFlow) were analyzed using an unpaired two-tailed Student's t test or one-way analysis of variance, and discrete variables (sex, substance usage, comorbidities, and intervention history) were compared using a two-tailed Fisher's exact test. All statistical analyses were conducted using STATA statistics/Data Analysis 8.0 (Stata Corporation, College Station, TX, USA).

\section{Results}

Table 1 summarizes the 30 patients including sex, age, comorbidities, dominant symptoms, target leg, Clinical-Etiology-Anatomy-Pathophysiology (CEAP) classification, and wound location. The mean age of the patients was $58.67 \pm 11.98$ years, and the majority of the patients were women $(24 / 30,80 \%)$. The dominant symptoms requiring surgical consultation were claudication $(13 / 30,43 \%)$, calf cramping $(5 / 30,17 \%)$, and static leg ulcers $(12 / 30,40 \%)$. The left leg was the target leg requiring treatment in most patients $(20 / 30,67 \%)$. Moreover, most patients were scheduled to undergo left superficial venous interventions, and all patients had lesions over $\mathrm{C} 4$. All patients exhibited saphenofemoral junction insufficiency in the Duplex study and morphological features of varicose veins in preoperative TRANCE-MRI. 
Table 1. Demographic data of the 30 patients with symptomatic varicose vein TRANCE MR as preoperative evaluation.

\begin{tabular}{|c|c|c|c|c|c|c|c|c|c|c|}
\hline No & Age & Sex & Comorbidities & $\begin{array}{l}\text { Treating } \\
\text { Legs }\end{array}$ & Symptoms & $\begin{array}{l}\text { C in } \\
\text { CEAP }\end{array}$ & $\begin{array}{l}E \text { in } \\
\text { CEAP }\end{array}$ & $A$ in CEAP & $\begin{array}{l}P \text { in } \\
\text { CEAP }\end{array}$ & $\begin{array}{l}\text { Wound } \\
\text { Location }\end{array}$ \\
\hline 1 & 46 & $\mathrm{~F}$ & Nil & Left & Claudication & $\mathrm{C} 4 \mathrm{~b}$ & Ep & $\begin{array}{c}\text { GSVa, GSVb, } \\
\text { ASV }\end{array}$ & $\operatorname{Pr}$ & no \\
\hline 2 & 46 & F & Nil & Left & Claudication & $\mathrm{C} 5$ & Ep & GSVa, GSVb & $\operatorname{Pr}$ & no \\
\hline 3 & 58 & $\mathrm{~F}$ & Nil & Left & Claudication & $\mathrm{C} 4 \mathrm{a}$ & Ep & GSVa, GSVb & $\operatorname{Pr}$ & no \\
\hline 4 & 82 & $\mathrm{~F}$ & Nil & Left & Claudication & $\mathrm{C} 4 \mathrm{c}$ & Ep & $\begin{array}{c}\text { GSVa, GSVb, } \\
\text { SSV }\end{array}$ & $\operatorname{Pr}$ & no \\
\hline 5 & 59 & F & HTN & Left & Claudication & $C 4 b$ & Ep & GSVa, GSVb & $\operatorname{Pr}$ & no \\
\hline 6 & 84 & $\mathrm{~F}$ & $\begin{array}{c}\text { Severe MR and } \\
\text { TR, CHF }\end{array}$ & Left & $\begin{array}{c}\text { calves } \\
\text { cramping }\end{array}$ & $\mathrm{C} 4 \mathrm{c}$ & Ep & GSVa, GSVb & $\operatorname{Pr}$ & no \\
\hline 7 & 58 & M & $\mathrm{Nl}$ & Left & Claudication & C5 & Ep & GSVa, GSVb & $\operatorname{Pr}$ & no \\
\hline 8 & 57 & $\mathrm{~F}$ & Nil & Right & Claudication & C5 & Ep & GSVa, GSVb & $\operatorname{Pr}$ & no \\
\hline 9 & 65 & $\mathrm{~F}$ & HTN & Left & $\begin{array}{c}\text { calves } \\
\text { cramping }\end{array}$ & C5 & Ep & $\begin{array}{c}\text { GSVa, GSVb, } \\
\text { CPV }\end{array}$ & $\operatorname{Pr}$ & no \\
\hline 10 & 53 & $\mathrm{~F}$ & Nil & Left & $\begin{array}{l}\text { calves } \\
\text { cramping }\end{array}$ & $\mathrm{C} 4 \mathrm{a}$ & Ep & $\begin{array}{c}\text { GSVa, GSVb, } \\
\text { CPV }\end{array}$ & $\operatorname{Pr}$ & no \\
\hline 11 & 56 & $\mathrm{~F}$ & Nil & Right & Wound & C5 & Ep & $\begin{array}{l}\text { GSVa, GSVb, } \\
\text { SSV, CPV }\end{array}$ & $\operatorname{Pr}$ & $\begin{array}{l}\text { medial } \\
\text { ankle }\end{array}$ \\
\hline 12 & 43 & M & Nil & Left & Wound & C5 & Ep & GSVa, GSVb & $\operatorname{Pr}$ & no \\
\hline 13 & 59 & $\mathrm{~F}$ & Nil & Left & Claudication & $\mathrm{C} 4 \mathrm{~b}$ & Ep & $\begin{array}{c}\text { GSVa, GSVb, } \\
\text { SSV, Vein of } \\
\text { Giacomini }\end{array}$ & $\operatorname{Pr}$ & no \\
\hline 14 & 55 & $\mathrm{~F}$ & HTN & Left & Claudication & $\mathrm{C} 4 \mathrm{a}$ & Ep & $\begin{array}{c}\text { GSVa, GSVb, } \\
\text { CPV }\end{array}$ & $\operatorname{Pr}$ & no \\
\hline 15 & 69 & $\mathrm{~F}$ & HTN & Right & Claudication & $\mathrm{C} 4 \mathrm{a}$ & Ep & $\begin{array}{c}\text { GSVa, GSVb, } \\
\text { CPV }\end{array}$ & $\operatorname{Pr}$ & no \\
\hline 16 & 67 & $\mathrm{~F}$ & nil & Left & Claudication & $\mathrm{C} 4 \mathrm{c}$ & Ep & $\begin{array}{l}\text { GSVa, GSVb, } \\
\text { SSV, CPV }\end{array}$ & $\operatorname{Pr}$ & no \\
\hline 17 & 71 & $\mathrm{~F}$ & Nil & Left & Claudication & $\mathrm{C} 4 \mathrm{~b}$ & Ep & $\begin{array}{c}\text { GSVa, GSVb, } \\
\text { CPV }\end{array}$ & $\operatorname{Pr}$ & no \\
\hline 18 & 38 & $\mathrm{~F}$ & Nil & Right & Claudication & $\mathrm{C} 4 \mathrm{c}$ & Ep & $\begin{array}{c}\text { GSVb, SSV, } \\
\text { TPV }\end{array}$ & $\operatorname{Pr}$ & no \\
\hline 19 & 59 & $\mathrm{~F}$ & $\mathrm{DM}$ & Left & Wound & C6 & Ep & $\begin{array}{l}\text { GSVa, GSVb, } \\
\text { SSV, CPV }\end{array}$ & $\operatorname{Pr}$ & $\begin{array}{l}\text { gaiter } \\
\text { area }\end{array}$ \\
\hline 20 & 68 & F & Nil & Right & Wound & C6r & Ep & $\begin{array}{c}\text { GSVa, GSVb, } \\
\text { CPV }\end{array}$ & $\operatorname{Pr}$ & $\begin{array}{l}\text { medial } \\
\text { ankle }\end{array}$ \\
\hline 21 & 58 & F & Nil & Right & Wound & C6 & Ep & $\begin{array}{c}\text { GSVa, GSVb, } \\
\text { CPV }\end{array}$ & $\operatorname{Pr}$ & $\begin{array}{l}\text { medial } \\
\text { ankle }\end{array}$ \\
\hline 22 & 40 & M & Nil & Left & Wound & C6 & Ep & GSVa, GSVb & $\operatorname{Pr}$ & $\begin{array}{l}\text { medial } \\
\text { ankle }\end{array}$ \\
\hline 23 & 43 & M & Obese & Left & Wound & C6r & Ep & $\begin{array}{l}\text { GSVa, GSVb, } \\
\text { SSV, CPV }\end{array}$ & $\operatorname{Pr}$ & $\begin{array}{l}\text { lateral } \\
\text { malleola }\end{array}$ \\
\hline 24 & 53 & $\mathrm{~F}$ & Nil & Right & Wound & C6 & Ep & $\begin{array}{c}\text { GSVa, GSVb, } \\
\text { SSV }\end{array}$ & $\operatorname{Pr}$ & $\begin{array}{l}\text { medial } \\
\text { ankle }\end{array}$ \\
\hline 25 & 50 & M & Nil & Right & Wound & C6 & Ep & GSVa, GSVb & $\operatorname{Pr}$ & $\begin{array}{l}\text { medial } \\
\text { ankle }\end{array}$ \\
\hline 26 & 82 & $\mathrm{~F}$ & Nil & Right & Wound & C6r & Ep & GSVa, GSVb & $\operatorname{Pr}$ & $\begin{array}{l}\text { medial } \\
\text { ankle }\end{array}$ \\
\hline 27 & 67 & M & CVA, HTN, DM & Left & Wound & C6 & Ep & $\begin{array}{c}\text { GSVa, GSVb, } \\
\text { SSV }\end{array}$ & $\operatorname{Pr}$ & $\begin{array}{l}\text { medial } \\
\text { ankle }\end{array}$ \\
\hline
\end{tabular}


Table 1. Cont.

\begin{tabular}{|c|c|c|c|c|c|c|c|c|c|c|}
\hline No & Age & Sex & Comorbidities & $\begin{array}{c}\text { Treating } \\
\text { Legs }\end{array}$ & Symptoms & $\begin{array}{l}C \text { in } \\
\text { CEAP }\end{array}$ & $\begin{array}{l}E \text { in } \\
\text { CEAP }\end{array}$ & $A$ in CEAP & $\begin{array}{l}P \text { in } \\
\text { CEAP }\end{array}$ & $\begin{array}{l}\text { Wound } \\
\text { Location }\end{array}$ \\
\hline 28 & 58 & $\mathrm{~F}$ & Nil & Left & Wound & C6r & Ep & GSVa, GSVb & $\operatorname{Pr}$ & $\begin{array}{l}\text { medial } \\
\text { ankle }\end{array}$ \\
\hline 29 & 54 & $\mathrm{~F}$ & Nil & Left & $\begin{array}{l}\text { calves } \\
\text { cramping }\end{array}$ & $\mathrm{C} 5$ & Ep & GSVa, GSVb & $\operatorname{Pr}$ & no \\
\hline 30 & 62 & $\mathrm{~F}$ & Nil & Right & $\begin{array}{l}\text { calves } \\
\text { cramping }\end{array}$ & $\mathrm{C} 5$ & Ep & $\begin{array}{c}\text { GSVa, GSVb, } \\
\text { SSV }\end{array}$ & $\operatorname{Pr}$ & no \\
\hline
\end{tabular}

AASV, anterior accessory saphenous vein; CEAP, Clinical-Etiology-Anatomy-Pathophysiology; CHF, congestive heart failure; CPV, calf perforator vein; CVA, cerebral vascular accident; DM, diabetes mellitus; F, female; HTN, hypertension; GSVa, great saphenous vein above knee; GSVb, great saphenous vein below knee; M, male; MR, mitral regurgitation; SSV, short saphenous vein; TPV, thigh perforator vein.

Table 2 summarizes the interventional data of the 30 patients. In addition to the standard truncal ablation of the GSV, six patients $(6 / 30,20 \%)$ received a second ablation according to TRANCE-MRI mapping for the accessory saphenous vein, posterior accessory saphenous vein (vein of Giacomini), small saphenous veins, and bifurcated GSVs. Varicose veins over $1 \mathrm{~cm}$ in diameter on TRANCE-MRI were removed by creating a small incision; veins less than $1 \mathrm{~cm}$ in diameter were treated through sclerotherapy.

Table 2. Interventional data of the 25 patients with symptomatic varicose vein TRANCE MR as preoperative evaluation.

\begin{tabular}{|c|c|c|c|c|c|c|c|c|c|}
\hline $\begin{array}{c}\text { Patient } \\
\text { No }\end{array}$ & Device & $\begin{array}{l}\text { Primary } \\
\text { Ablation }\end{array}$ & $\begin{array}{l}\text { Secondary } \\
\text { Ablation }\end{array}$ & $\begin{array}{l}\text { Thigh } \\
\text { Cut- } \\
\text { down }\end{array}$ & $\begin{array}{l}\text { Groin } \\
\text { Cut- } \\
\text { down }\end{array}$ & Sclerotherapy & Phlebectomy & $\begin{array}{c}\text { Tumescent } \\
\text { Solution } \\
\text { Use }\end{array}$ & Complication \\
\hline 1 & $\begin{array}{l}\text { VNUS } \\
\text { (metronic) }\end{array}$ & LGSV & ASV & Yes & Nil & Calf (alcohol) & $\begin{array}{l}\text { Calf and } \\
\text { knee }\end{array}$ & Yes & Nil \\
\hline 2 & $\begin{array}{l}\text { Atoven } \\
\text { catheter }\end{array}$ & LGSV & Nil & Nil & Yes & $\begin{array}{c}\text { Calf } \\
\text { (Fibrovein) }\end{array}$ & Calf & Yes & Echymosis \\
\hline 3 & $\begin{array}{l}\text { Atoven } \\
\text { catheter }\end{array}$ & LGSV & Nil & Yes & Nil & Nil & Nil & Yes & Nil \\
\hline 4 & Venaseal & LGSV & SSV & Yes & Nil & Nil & Nil & Nil & Nil \\
\hline 5 & $\begin{array}{l}\text { Atoven } \\
\text { catheter }\end{array}$ & LGSV & Nil & Yes & Nil & Calf(Fibrovein) & Nil & Yes & Nil \\
\hline 6 & Venaseal & LGSV & Nil & Nil & Nil & Nil & Nil & Nil & Nil \\
\hline 7 & $\begin{array}{l}\text { Atoven } \\
\text { catheter }\end{array}$ & LGSV & Nil & Nil & Nil & Calf(Fibrovein) & Nil & Yes & Nil \\
\hline 8 & Venaseal & RGSV & Nil & Yes & Yes & Nil & Nil & Nil & Nil \\
\hline 9 & $\begin{array}{l}\text { Atoven } \\
\text { catheter }\end{array}$ & LGSV & LSV & Nil & Nil & Calf(Fibrovein) & Nil & Nil & Nil \\
\hline 10 & $\begin{array}{l}\text { A.R.C } \\
\text { catheter }\end{array}$ & LGSV & Nil & Nil & Nil & Calf(Fibrovein) & $\begin{array}{l}\text { popliteal } \\
\text { fossa }\end{array}$ & Yes & Nil \\
\hline 11 & $\begin{array}{l}\text { Atoven } \\
\text { catheter }\end{array}$ & LGSV & Nil & Nil & Nil & Calf(Fibrovein) & Nil & Yes & Nil \\
\hline 12 & $\begin{array}{l}\text { A.R.C } \\
\text { catheter }\end{array}$ & LGSV & Nil & Nil & Nil & Calf(Fibrovein) & Nil & Yes & Nil \\
\hline 13 & $\begin{array}{l}\text { A.R.C } \\
\text { catheter }\end{array}$ & LGSV & $\begin{array}{l}\text { SSV and } \\
\text { PASV }\end{array}$ & Nil & Nil & Calf(Fibrovein) & Nil & Yes & Nil \\
\hline 14 & Venaseal & LGSV & Nil & Nil & Nil & Nil & Nil & Nil & Nil \\
\hline 15 & $\begin{array}{l}\text { Atoven } \\
\text { catheter }\end{array}$ & RGSV & Nil & Nil & Nil & Calf (alcohol) & Nil & Yes & Nil \\
\hline 16 & Venaseal & LGSV & Nil & Nil & Nil & Calf(Fibrovein) & Nil & Nil & Nil \\
\hline 17 & $\begin{array}{l}\text { A.R.C } \\
\text { catheter }\end{array}$ & LGSV & $\begin{array}{l}\text { bifurcated } \\
\text { GSV }\end{array}$ & Nil & Nil & Nil & Nil & Yes & Nil \\
\hline 18 & $\begin{array}{l}\text { A.R.C } \\
\text { catheter }\end{array}$ & RGSV & Nil & Nil & Nil & $\begin{array}{c}\text { lateral } \\
\text { thigh(Fibrovein) }\end{array}$ & Nil & Yes & Nil \\
\hline 19 & $\begin{array}{l}\text { Atoven } \\
\text { catheter }\end{array}$ & LGSV & Nil & Yes & Nil & Calf(Fibrovein) & $\begin{array}{l}\text { Calf and } \\
\text { knee }\end{array}$ & Yes & Nil \\
\hline
\end{tabular}


Table 2. Cont

\begin{tabular}{|c|c|c|c|c|c|c|c|c|c|}
\hline $\begin{array}{c}\text { Patient } \\
\text { No }\end{array}$ & Device & $\begin{array}{l}\text { Primary } \\
\text { Ablation }\end{array}$ & $\begin{array}{l}\text { Secondary } \\
\text { Ablation }\end{array}$ & $\begin{array}{l}\text { Thigh } \\
\text { Cut- } \\
\text { down }\end{array}$ & $\begin{array}{l}\text { Groin } \\
\text { Cut- } \\
\text { down }\end{array}$ & Sclerotherapy & Phlebectomy & $\begin{array}{l}\text { Tumescent } \\
\text { Solution } \\
\text { Use }\end{array}$ & Complication \\
\hline 20 & Venaseal & RGSV & Nil & Nil & Nil & Nil & Nil & Nil & topical allergy \\
\hline 21 & $\begin{array}{l}\text { Atoven } \\
\text { catheter }\end{array}$ & RGSV & $\begin{array}{c}\text { bifurcated } \\
\text { GSV }\end{array}$ & Nil & Nil & Calf(Fibrovein) & Nil & Yes & Nil \\
\hline 22 & Venaseal & LGSV & Nil & Nil & Nil & Nil & Nil & Nil & Nil \\
\hline 23 & $\begin{array}{l}\text { A.R.C } \\
\text { catheter }\end{array}$ & LGSV & LSSV & Nil & Nil & Calf(Fibrovein) & Nil & Yes & Nil \\
\hline 24 & $\begin{array}{c}\text { A.R.C } \\
\text { catheter }\end{array}$ & RGSV & Nil & Nil & Nil & Calf(Fibrovein) & Nil & Yes & Nil \\
\hline 25 & $\begin{array}{l}\text { A.R.C } \\
\text { catheter }\end{array}$ & RGSV & Nil & Nil & Nil & Calf(Fibrovein) & Nil & Yes & Nil \\
\hline 26 & Venaseal & RGSV & Nil & Nil & Nil & Nil & Nil & Nil & Nil \\
\hline 27 & $\begin{array}{l}\text { A.R.C } \\
\text { catheter }\end{array}$ & LGSV & SSV & Nil & Nil & Calf(Fibrovein) & Nil & Yes & Nil \\
\hline 28 & $\begin{array}{l}\text { A.R.C } \\
\text { catheter }\end{array}$ & LGSV & Nil & Nil & Nil & Nil & Nil & Yes & Nil \\
\hline 29 & $\begin{array}{l}\text { A.R.C } \\
\text { catheter }\end{array}$ & LGSV & Nil & Nil & Nil & & & Yes & Nil \\
\hline 30 & $\begin{array}{l}\text { Atoven } \\
\text { catheter }\end{array}$ & RGSV & Nil & Nil & Nil & Nil & Nil & Yes & Nil \\
\hline
\end{tabular}

LGSV: left saphenous vein; RGSV: right saphenous vein.

\subsection{Comparison between Duplex Scanning and TRANCE-MRI Preoperatively}

All 30 patients underwent preoperative duplex scanning and TRANCE-MRI preoperatively. TRANCE-MRI and Duplex identically excluded patients with DVT (Table 3). The TRANCE-MRI criterion of superficial venous reflux (QFlow mean flux (MF) ratio of GSV /PV > 1) was compared with the duplex scan (the gold standard to examine the saphenous femoral junction reflux) with regard to their abilities to detect superficial venous reflux by using a Cohen's kappa coefficient of 0.967 [10].

Table 3. Diagnostic tool performance for venous disease in these 30 patients.

\begin{tabular}{|c|c|c|c|c|c|c|c|}
\hline No & $\begin{array}{l}\text { Dopplex- } \\
\text { DVT }\end{array}$ & $\begin{array}{l}\text { Dupplex: SFJ } \\
\text { Reflux }\end{array}$ & $\begin{array}{l}\text { Dupplex: } \\
\text { Additional } \\
\text { Target for } \\
\text { Ablation }\end{array}$ & $\begin{array}{l}\text { TRANCE- } \\
\text { DVT }\end{array}$ & $\begin{array}{c}\text { TRANCE MR } \\
\text { GSV/PV MF } \\
\text { QFlow >1 }\end{array}$ & $\begin{array}{l}\text { TRANCE- } \\
\text { MTS Like } \\
\text { Lesion }\end{array}$ & $\begin{array}{l}\text { TRANCE- } \\
\text { Additional } \\
\text { Target for } \\
\text { Ablation }\end{array}$ \\
\hline 1 & No & Yes & No & No & Yes & No & Yes (ASV) \\
\hline 2 & No & Yes & No & No & Yes & No & No \\
\hline 3 & No & Yes & No & No & Yes & No & No \\
\hline 4 & No & Yes & No & No & Yes & No & SSV \\
\hline 5 & No & Yes & No & No & Yes & No & No \\
\hline 6 & No & Yes & No & No & Yes & No & No \\
\hline 7 & No & Yes & No & No & Yes & No & No \\
\hline 8 & No & Yes & No & No & Yes & No & No \\
\hline 9 & No & Yes & No & No & Yes & No & LSV \\
\hline 10 & No & Yes & No & No & Yes & No & No \\
\hline 11 & No & Yes & No & No & Yes & No & No \\
\hline 12 & No & Yes & No & No & Yes & No & No \\
\hline 13 & No & Yes & No & No & Yes & No & $\begin{array}{c}\text { Yes (SSV and } \\
\text { PASV) }\end{array}$ \\
\hline 14 & No & Yes & No & No & Yes & No & No \\
\hline 15 & No & Yes & No & No & Yes & No & No \\
\hline 16 & No & Yes & No & No & Yes & No & No \\
\hline 17 & No & Yes & No & No & Yes & No & $\begin{array}{c}\text { Yes (bifurcated } \\
\text { GSV) }\end{array}$ \\
\hline
\end{tabular}


Table 3. Cont.

\begin{tabular}{|c|c|c|c|c|c|c|c|}
\hline No & $\begin{array}{l}\text { Dopplex- } \\
\text { DVT }\end{array}$ & $\begin{array}{l}\text { Dupplex: SFJ } \\
\text { Reflux }\end{array}$ & $\begin{array}{l}\text { Dupplex: } \\
\text { Additional } \\
\text { Target for } \\
\text { Ablation }\end{array}$ & $\begin{array}{l}\text { TRANCE- } \\
\text { DVT }\end{array}$ & $\begin{array}{c}\text { TRANCE MR } \\
\text { GSV/PV MF } \\
\text { QFlow >1 }\end{array}$ & $\begin{array}{l}\text { TRANCE- } \\
\text { MTS Like } \\
\text { Lesion }\end{array}$ & $\begin{array}{c}\text { TRANCE- } \\
\text { Additional } \\
\text { Target for } \\
\text { Ablation }\end{array}$ \\
\hline 18 & No & No & No & No & Yes & No & No \\
\hline 19 & No & Yes & No & No & Yes & No & No \\
\hline 20 & No & Yes & No & No & Yes & No & No \\
\hline 21 & No & Yes & No & No & Yes & No & $\begin{array}{c}\text { Yes (bifurcated } \\
\text { GSV) }\end{array}$ \\
\hline 22 & No & Yes & No & No & Yes & No & No \\
\hline 23 & No & Yes & No & No & Yes & Yes & Yes (LSSV) \\
\hline 24 & No & Yes & No & No & Yes & No & No \\
\hline 25 & No & Yes & No & No & Yes & No & No \\
\hline 26 & No & Yes & No & No & Yes & No & No \\
\hline 27 & No & Yes & No & No & Yes & Yes & Yes (LSSV) \\
\hline 28 & No & Yes & No & No & Yes & No & No \\
\hline 29 & No & Yes & No & No & Yes & No & No \\
\hline 30 & No & Yes & No & No & Yes & No & No \\
\hline
\end{tabular}

ASV, accessory saphneous vein; DVT, deep venou thrombosis; LSSV, left short saphenous vein; MF, mean flux; MTS, May-Thurner Syndrome; PASV, posterior accessory saphenous vein; SFJ, sphano-femoral junction.

\subsection{Comparison of TRANCE-MRI Hemodynamic Parameters between the Morbid Limbs and Healthy Volunteers}

QFlow analysis performed through TRANCE-MRI examined the SV ( $\mathrm{mL})$, forward flow volume $(\mathrm{FFV}, \mathrm{mL}), \mathrm{MF}(\mathrm{mL}), \mathrm{SD}(\mathrm{cm})$, and $\mathrm{MV}(\mathrm{cm})$ in the vena cava, EIVs, FVs, PVs, and GSVs in the 30 patients and healthy controls. To decrease bias in the QFlow analysis, we analyzed the same side of the legs of controls as that of patients who received interventions. Table 4 shows the findings of the QFlow comparison of 10 patients who received interventions in their left leg with the left legs of 20 controls. SVs were higher in the EIVs $(p=0.021)$ in the left-leg-intervention group. The MF was higher in the EIV ( $p=$ $0.012)$ and tended to increase in the GSV $(p=0.087)$ in the left-leg-intervention group. SD was longer in EIV segments.

Table 4. Comparison of the QFlow parameters between the left legs of health controls and the left legs planning for truncal ablation.

\begin{tabular}{|c|c|c|c|c|c|c|c|c|c|}
\hline 0 & & \multicolumn{2}{|c|}{$\begin{array}{l}\text { Health Volunteers } \\
\qquad(\mathrm{N}=\mathbf{1 0})\end{array}$} & \multicolumn{2}{|c|}{$\begin{array}{c}\text { Planned Superficial } \\
\text { Intervention }(\mathbf{N}=\mathbf{2 0})\end{array}$} & & \multicolumn{3}{|c|}{ Power Analysis } \\
\hline QFlow & Segments & Mean & $\begin{array}{l}\text { Standard } \\
\text { Deviation }\end{array}$ & Mean & $\begin{array}{l}\text { Standard } \\
\text { Deviation }\end{array}$ & $p$ Value & Power & $\begin{array}{l}\text { Effect } \\
\text { Size d }\end{array}$ & $\begin{array}{c}\text { Total } \\
\text { Sample } \\
\text { Size }\end{array}$ \\
\hline \multicolumn{10}{|c|}{ SV (Stroke Volumes) } \\
\hline & IVC & 18.538 & 6.125 & 16.147 & 6.135 & 0.349 & 0.458 & 0.390 & 186 \\
\hline & LEIV & 3.691 & 1.050 & 5.056 & 1.838 & $0.021 *$ & 0.768 & 0.912 & 36 \\
\hline & LFV & 1.202 & 0.746 & 1.838 & 1.417 & 0.202 & 0.573 & 0.561 & 90 \\
\hline & LGSV & 0.459 & 0.324 & 1.063 & 1.145 & 0.118 & 0.668 & 0.718 & 56 \\
\hline & LPV & 0.643 & 0.332 & 1.112 & 1.324 & 0.285 & 0.524 & & \\
\hline \multicolumn{10}{|c|}{$\begin{array}{c}\text { FFV (Foreward Flow } \\
\text { Volumes) }\end{array}$} \\
\hline & IVC & 18.992 & 6.192 & 16.887 & 6.110 & 0.410 & 0.524 & 0.486 & 120 \\
\hline & LEIV & 3.849 & 1.114 & 5.433 & 2.688 & 0.090 & 0.697 & 0.770 & 50 \\
\hline & LFV & 1.230 & 0.719 & 1.855 & 1.472 & 0.223 & 0.559 & 0.539 & 98 \\
\hline & LGSV & 0.473 & 0.308 & 0.834 & 0.662 & 0.119 & 0.657 & 0.698 & 60 \\
\hline & LPV & 0.654 & 0.317 & 1.144 & 1.409 & 0.293 & 0.519 & 0.479 & 124 \\
\hline
\end{tabular}


Table 4. Cont.

\begin{tabular}{|c|c|c|c|c|c|c|c|c|c|}
\hline \multirow{2}{*}{$\begin{array}{c}0 \\
\text { QFlow }\end{array}$} & \multirow[b]{2}{*}{ Segments } & \multicolumn{2}{|c|}{$\begin{array}{l}\text { Health Volunteers } \\
\qquad(\mathrm{N}=10)\end{array}$} & \multicolumn{2}{|c|}{$\begin{array}{c}\text { Planned Superficial } \\
\text { Intervention }(\mathbf{N}=\mathbf{2 0})\end{array}$} & \multirow[b]{2}{*}{$p$ Value } & \multicolumn{3}{|c|}{ Power Analysis } \\
\hline & & Mean & $\begin{array}{l}\text { Standard } \\
\text { Deviation }\end{array}$ & Mean & $\begin{array}{l}\text { Standard } \\
\text { Deviation }\end{array}$ & & Power & $\begin{array}{l}\text { Effect } \\
\text { Size d }\end{array}$ & $\begin{array}{c}\text { Total } \\
\text { Sample } \\
\text { Size }\end{array}$ \\
\hline \multicolumn{10}{|c|}{$\begin{array}{l}\text { BFV (Backward Flow } \\
\text { Volumes) }\end{array}$} \\
\hline & IVC & 0.452 & 1.028 & 0.738 & 2.243 & 0.711 & 0.303 & 0.164 & 1038 \\
\hline & LEIV & 0.155 & 0.240 & 0.375 & 1.412 & 0.632 & 0.339 & 0.218 & 590 \\
\hline & LFV & 0.027 & 0.049 & 0.017 & 0.061 & 0.644 & 0.321 & 0.191 & 764 \\
\hline & LGSV & 0.012 & 0.022 & 0.333 & 1.319 & 0.453 & 0.393 & 0.344 & 212 \\
\hline & LPV & 0.009 & 0.020 & 0.030 & 0.095 & 0.502 & 0.370 & 0.306 & 266 \\
\hline \multicolumn{10}{|c|}{$\begin{array}{l}\text { RF (Regurgitant } \\
\text { Fraction) }\end{array}$} \\
\hline & IVC & 2.186 & 4.892 & 3.963 & 13.140 & 0.688 & 0.426 & 0.344 & 238 \\
\hline & LEIV & 3.749 & 7.477 & 3.230 & 10.679 & 0.888 & 0.233 & 0.056 & 8780 \\
\hline & LFV & 5.206 & 5.852 & 0.629 & 1.877 & 0.168 & 0.826 & 1.053 & 28 \\
\hline & LGSV & 9.650 & 26.954 & 3.924 & 7.372 & 0.296 & 0.389 & 0.290 & 334 \\
\hline & Lt PV & 5.986 & 8.540 & 5.291 & 17.618 & 0.917 & 0.230 & 0.050 & 11048 \\
\hline \multicolumn{10}{|c|}{$\begin{array}{c}\text { ASV (Absolute Stroke } \\
\text { Volumes) }\end{array}$} \\
\hline & IVC & 19.448 & 6.426 & 17.627 & 6.864 & 0.512 & 0.378 & 0.274 & 374 \\
\hline & LEIV & 4.008 & 1.222 & 5.808 & 3.881 & 0.169 & 0.614 & 0.626 & 74 \\
\hline & LFV & 1.262 & 0.695 & 1.872 & 1.528 & 0.247 & 0.543 & 0.514 & 108 \\
\hline & LGSV & 0.487 & 0.294 & 1.169 & 1.454 & 0.158 & 0.629 & 0.651 & 68 \\
\hline & LPV & 0.665 & 0.303 & 1.174 & 1.495 & 0.301 & 0.514 & 0.472 & 128 \\
\hline \multicolumn{10}{|c|}{ MF (Mean Flux) } \\
\hline & IVC & 21.336 & 6.848 & 18.679 & 7.912 & 0.395 & 0.437 & 0.359 & 218 \\
\hline & LEIV & 3.798 & 0.871 & 5.395 & 2.136 & $0.012 *$ & 0.797 & 0.979 & 32 \\
\hline & LFV & 1.246 & 0.776 & 1.924 & 1.268 & 0.140 & 0.626 & 0.645 & 70 \\
\hline & LGSV & 0.477 & 0.362 & 1.097 & 1.057 & 0.087 & 0.705 & 0.784 & 48 \\
\hline & LPV & 0.650 & 0.322 & 1.140 & 1.184 & 0.215 & 0.576 & 0.565 & 90 \\
\hline \multicolumn{10}{|c|}{ SD (Stroke Distance) } \\
\hline & IVC & 9.519 & 3.317 & 11.026 & 5.368 & 0.438 & 0.422 & 0.338 & 246 \\
\hline & LEIV & 3.459 & 0.590 & 6.705 & 5.122 & 0.019 * & 0.758 & 0.890 & 38 \\
\hline & LFV & 4.092 & 3.357 & 4.751 & 3.008 & 0.603 & 0.331 & 0.207 & 652 \\
\hline & LGSV & 2.005 & 1.520 & 2.672 & 4.919 & 0.682 & 0.315 & 0.183 & 832 \\
\hline & LPV & 1.408 & 1.124 & 1.384 & 0.883 & 0.952 & 0.214 & 0.024 & 49768 \\
\hline \multicolumn{10}{|c|}{ MV (Mean Velocity) } \\
\hline & IVC & 11.169 & 4.354 & 12.304 & 4.975 & 0.563 & 0.356 & 0.243 & 474 \\
\hline & LEIV & 33.816 & 94.936 & 7.125 & 5.424 & 0.397 & 0.463 & 0.397 & 178 \\
\hline & LFV & 4.347 & 3.893 & 5.079 & 3.249 & 0.604 & 0.330 & 0.204 & 670 \\
\hline & LGSV & 2.033 & 1.554 & 2.954 & 4.973 & 0.577 & 0.361 & 0.250 & 448 \\
\hline & LPV & 1.458 & 1.252 & 1.452 & 0.886 & 0.989 & 0.203 & 0.005 & $>10,000$ \\
\hline
\end{tabular}

IVC, inferior vena cava; LEIA, left external iliac vein, LFV, left femoral vein; LGSV, left great saphaneous vein; LPV, left popliteal vein.

Table 5 shows the findings of the QFlow comparison of 10 patients who received intervention in their right leg with the right legs of 10 controls. SV $(p=0.002)$, FFV $(p=0.001)$, and MF ( $p=0.001)$ in the GSV were higher in the right-leg-intervention group. 
Table 5. Comparison of the QFlow parameters between the right legs of health controls and the right legs planning for truncal ablation.

\begin{tabular}{|c|c|c|c|c|c|c|c|c|c|}
\hline \multirow[b]{2}{*}{ QFlow } & \multirow[b]{2}{*}{ Segments } & \multicolumn{2}{|c|}{$\begin{array}{l}\text { Health Volunteers } \\
\qquad(\mathrm{N}=10)\end{array}$} & \multicolumn{2}{|c|}{$\begin{array}{l}\text { Planned Superficial } \\
\text { Intervention }(\mathbf{N}=10)\end{array}$} & \multirow[b]{2}{*}{$p$-Value } & \multicolumn{3}{|c|}{ Power Analysis } \\
\hline & & Mean & $\begin{array}{l}\text { Standard } \\
\text { Deviation }\end{array}$ & Mean & $\begin{array}{l}\text { Standard } \\
\text { Deviation }\end{array}$ & & Power & $\begin{array}{l}\text { Effect } \\
\text { Size d }\end{array}$ & $\begin{array}{c}\text { Total } \\
\text { Sample } \\
\text { Size }\end{array}$ \\
\hline \multicolumn{10}{|c|}{ SV (Stroke Volumes) } \\
\hline & IVC & 18.538 & 6.125 & 13.933 & 5.537 & 0.118 & 0.645 & 0.789 & 42 \\
\hline & REIV & 4.303 & 0.872 & 5.456 & 2.725 & 0.282 & 0.527 & 0.570 & 78 \\
\hline & RFV & 1.437 & 0.704 & 1.764 & 0.996 & 0.427 & 0.414 & 0.379 & 174 \\
\hline & RGSV & 0.360 & 0.265 & 0.893 & 0.342 & $0.002 *$ & 0.934 & 1.742 & 10 \\
\hline & Rt PV & 0.579 & 0.278 & 1.125 & 0.876 & 0.128 & 0.670 & 0.840 & 38 \\
\hline \multicolumn{10}{|c|}{$\begin{array}{c}\text { FFV (Foreward Flow } \\
\text { Volumes) }\end{array}$} \\
\hline & IVC & 18.992 & 6.192 & 14.594 & 5.395 & 0.133 & 0.670 & 0.840 & 38 \\
\hline & REIV & 4.605 & 1.074 & 5.625 & 2.624 & 0.278 & 0.491 & 0.509 & 98 \\
\hline & RFV & 1.451 & 0.691 & 1.774 & 0.985 & 0.426 & 0.414 & 0.379 & 174 \\
\hline & RGSV & 0.377 & 0.248 & 0.903 & 0.321 & $0.001 *$ & 0.945 & 1.832 & 10 \\
\hline & RPV & 0.600 & 0.277 & 1.144 & 0.864 & 0.125 & 0.674 & 0.848 & 36 \\
\hline \multicolumn{10}{|c|}{$\begin{array}{l}\text { BFV (Backward Flow } \\
\text { Volumes) }\end{array}$} \\
\hline & IVC & 0.452 & 1.028 & 0.659 & 1.102 & 0.687 & 0.305 & 0.194 & 660 \\
\hline & REIV & 0.299 & 0.396 & 0.166 & 0.216 & 0.380 & 0.436 & 0.416 & 146 \\
\hline & RFV & 0.012 & 0.025 & 0.009 & 0.025 & 0.788 & 0.267 & 0.128 & 1514 \\
\hline & RGSV & 0.026 & 0.046 & 0.009 & 0.025 & 0.351 & 0.468 & 0.470 & 114 \\
\hline & RPV & 0.018 & 0.030 & 0.016 & 0.024 & 0.896 & & & \\
\hline \multicolumn{10}{|c|}{$\begin{array}{l}\text { RF (Regurgitant } \\
\text { Fraction) }\end{array}$} \\
\hline & IVC & 2.186 & 4.892 & 4.755 & 7.635 & 0.398 & 0.232 & 0.062 & 6416 \\
\hline & REIV & 5.928 & 7.477 & 4.024 & 5.293 & 0.537 & 0.363 & 0.294 & 288 \\
\hline & RFV & 2.317 & 5.852 & 1.321 & 3.178 & 0.672 & 0.315 & 0.211 & 556 \\
\hline & RGSV & 15.705 & 26.954 & 2.731 & 7.725 & 0.176 & 0.574 & 0.654 & 60 \\
\hline & RPV & 4.737 & 8.540 & 4.115 & 6.736 & 0.869 & 0.242 & 0.081 & 3784 \\
\hline \multicolumn{10}{|c|}{$\begin{array}{c}\text { ASV (Absolute Stroke } \\
\text { Volumes) }\end{array}$} \\
\hline & IVC & 19.448 & 6.426 & 15.258 & 5.474 & 0.162 & 0.600 & 0.702 & 52 \\
\hline & REIV & 4.908 & 1.367 & 5.781 & 2.544 & 0.364 & 0.443 & 0.428 & 138 \\
\hline & RFV & 1.464 & 0.681 & 1.784 & 0.973 & 0.424 & 0.415 & 0.381 & 172 \\
\hline & RGSV & 0.404 & 0.220 & 0.913 & 0.302 & 0.001 & 0.955 & 1.924 & 10 \\
\hline & RPV & 0.620 & 0.278 & 1.164 & 0.852 & 0.120 & 0.678 & 0.858 & 36 \\
\hline \multicolumn{10}{|c|}{ MF (Mean Flux) } \\
\hline & IVC & 21.336 & 6.848 & 16.453 & 7.724 & 0.174 & 0.582 & 0.669 & 58 \\
\hline & REIV & 4.478 & 0.846 & 6.363 & 3.774 & 0.142 & 0.593 & 0.689 & 54 \\
\hline & RFV & 1.471 & 0.753 & 2.048 & 1.293 & 0.253 & 0.512 & 0.545 & 86 \\
\hline & RGSV & 0.372 & 0.289 & 1.013 & 0.400 & 0.001 * & 0.946 & 1.834 & 10 \\
\hline & RPV & 0.584 & 0.268 & 1.348 & 1.200 & 0.118 & 0.688 & 0.878 & 34 \\
\hline \multicolumn{10}{|c|}{ SD (Stroke Distance) } \\
\hline & IVC & 9.519 & 3.317 & 7.770 & 3.355 & 0.285 & 0.500 & 0.524 & 92 \\
\hline & REIV & 3.862 & 1.000 & 5.474 & 1.619 & 0.019 * & 0.814 & 1.198 & 20 \\
\hline & RFV & 4.737 & 3.660 & 3.026 & 1.492 & 0.234 & 0.550 & 0.612 & 68 \\
\hline & RGSV & 1.901 & 1.681 & 3.156 & 2.340 & 0.204 & 0.553 & 0.616 & 68 \\
\hline & Rt PV & 1.055 & 0.450 & 1.413 & 1.089 & 0.357 & 0.444 & 0.429 & 136 \\
\hline
\end{tabular}


Table 5. Cont

\begin{tabular}{|c|c|c|c|c|c|c|c|c|c|}
\hline \multirow[b]{2}{*}{ QFlow } & \multirow[b]{2}{*}{ Segments } & \multicolumn{2}{|c|}{$\begin{array}{l}\text { Health Volunteers } \\
(\mathrm{N}=10)\end{array}$} & \multicolumn{2}{|c|}{$\begin{array}{c}\text { Planned Superficial } \\
\text { Intervention }(\mathbf{N}=10)\end{array}$} & \multirow[b]{2}{*}{$p$-Value } & \multicolumn{3}{|c|}{ Power Analysis } \\
\hline & & Mean & $\begin{array}{l}\text { Standard } \\
\text { Deviation }\end{array}$ & Mean & $\begin{array}{l}\text { Standard } \\
\text { Deviation }\end{array}$ & & Power & $\begin{array}{l}\text { Effect } \\
\text { Size d }\end{array}$ & $\begin{array}{c}\text { Total } \\
\text { Sample } \\
\text { Size }\end{array}$ \\
\hline \multicolumn{10}{|c|}{ MV ( Mean Velocity) } \\
\hline & IVC & 11.169 & 4.354 & 9.121 & 4.894 & 0.362 & 0.452 & 0.442 & 128 \\
\hline & REIV & 4.086 & 1.246 & 4.995 & 2.719 & 0.359 & 0.444 & 0.430 & 136 \\
\hline & RFV & 4.975 & 4.241 & 3.433 & 1.852 & 0.354 & 0.469 & 0.471 & 114 \\
\hline & RGSV & 1.920 & 1.706 & 3.475 & 2.569 & 0.143 & 0.605 & 0.713 & 52 \\
\hline & RPV & 1.064 & 0.393 & 1.639 & 1.362 & 0.281 & 0.528 & 0.573 & 78 \\
\hline
\end{tabular}

IVC: inferior vena cava; REIA: right external iliac vein; RFV: right femoral vein; RGSV: right great saphaneous vein; RPV: right popliteal vein.

\section{Discussion}

Superficial venous interventions for varicose veins in the lower extremities mainly include truncal ablation, phlebectomy, and sclerotherapy. Patients suspected to have venous reflux disease of the legs undergo air plethysmography and US (duplex) as the initiation of their therapy. US, a rapid tool, can provide additional information regarding active and gravitational refluxes in the standing position when performed by experienced operators. However, US is operator dependent and does not gain information regarding the pelvis. In many institutions, including ours, duplex scanning is exclusively performed in US centers and not performed by the same physician in the clinic; this requires additional communication between staff to gain sufficient surgical information. Meanwhile, pelvic status, including vessel compression and occult benign and malignant pathology, can only be excluded by using other objective diagnostic tools. Venography is historically considered the gold standard for the detection of DVT and other venous occlusive diseases. However, venography is an invasive procedure and cannot reveal varicose veins outside the drainage course of the contrast-medium injection site; thus, it is no longer included in the preoperative evaluation of superficial venous interventions. Intravenous US (IVUS) is an imaging tool used for diagnosing deep vein disease and is mostly used for guiding effective endovascular treatment in iliac and caval venous obstructive diseases [11,12]. However, IVUS is invasive and provides only the details inside the venous lumen without those of the superficial venous system. CT venography may be feasible for the exclusion of pulmonary embolism in patients with symptoms of DVT in the legs; however, CT venography still requires the injection of contrast medium into the morbid limb to achieve optimal venous imaging of the extremities; this procedure can harm the diseased limb [13].

Magnetic resonance angiography (MRA) techniques used for reconstructing vascular structures include time-of-flight (TOF), phase-contrast and electrocardiography (ECG)gated TSE MRA [11]. The major disadvantages of TOF-MRV are that the FOV is small for each image obtained and that it requires extraordinary time to gain a whole image of the legs. MRI with gadolinium-based contrast medium is a relatively rapid method for imaging the lower extremities [12,14]. Although MRI does not involve radiation exposure, noniodinated contrast agents used in the imaging process still produce undesirable effects. For example, nephrogenic sclerosing fibrosis is a severe complication of gadolinium-based contrast agents in patients with impairment of kidney function and may even occur in patients with normal renal function $[15,16]$. Phase-contrast MRI depends on phase shifts caused by blood flow. Thus, this technique permits the use of coronal or sagittal slice orientations with an FOV along the direction of the vessel of interest and can quantitatively measure the dynamic flow of the chosen region of interest. Most studies have used phase-contrast MRA for evaluating central nervous system pathologies including hydrocephalus $[17,18]$. An ECG-gated multistep TSE technique (i.e., TRANCE-MRI) enables the imaging of vessels in the whole lower extremity. ECG gating helps to adapt imaging times to different flow characteristics and therefore optimize image quality faster. Although some studies have used non-contrast-enhanced MRI, most have used this technique to 
evaluate arterial diseases [19-23]. Our team has innovated the use of TRANCE-MRI to provide more valuable information for the management of complicated lower venous diseases since 2017 [4-6,9]. The examination time could be shortened to less than $25 \mathrm{~min}$ by our experienced radiological teams with a reasonable cost (250 USD/each exam). The morphology of the venous anatomy of the lower extremities, especially the low-flow superficial venous system, could be clearly demonstrated through 3D imaging without the use of contrast medium or radiation. TRANCE-MRI has been the standard preoperative evaluation modality for superficial venous interventions in our institution and has positive feedback from the patients during the preoperative communication (Figure 2). We use duplex scanning to identify venous thrombosis and superficial venous reflux. TRANCE-MRI can be arranged to plan for further venous interventions. The pelvic status was proven no coexisted external compression inside the pelvis first. The morphology of the GSVs, accessory saphenous veins, and small saphenous veins is routinely examined and referred for anesthesia management in accordance with surgical plans (Supplementary Video S1) [11]. TRANCE-MRI reveals the tributaries of the calves in detail, enabling excellent communication with patients with regard to their treatment options such as sclerotherapy and phlebectomy. Our previous study indicated that a TRANCE-MRI GSV/PV ratio of $>1$ may be a hallmark of superficial venous reflux [10]. These signs were correlated to the duplex findings of these 30 patients.

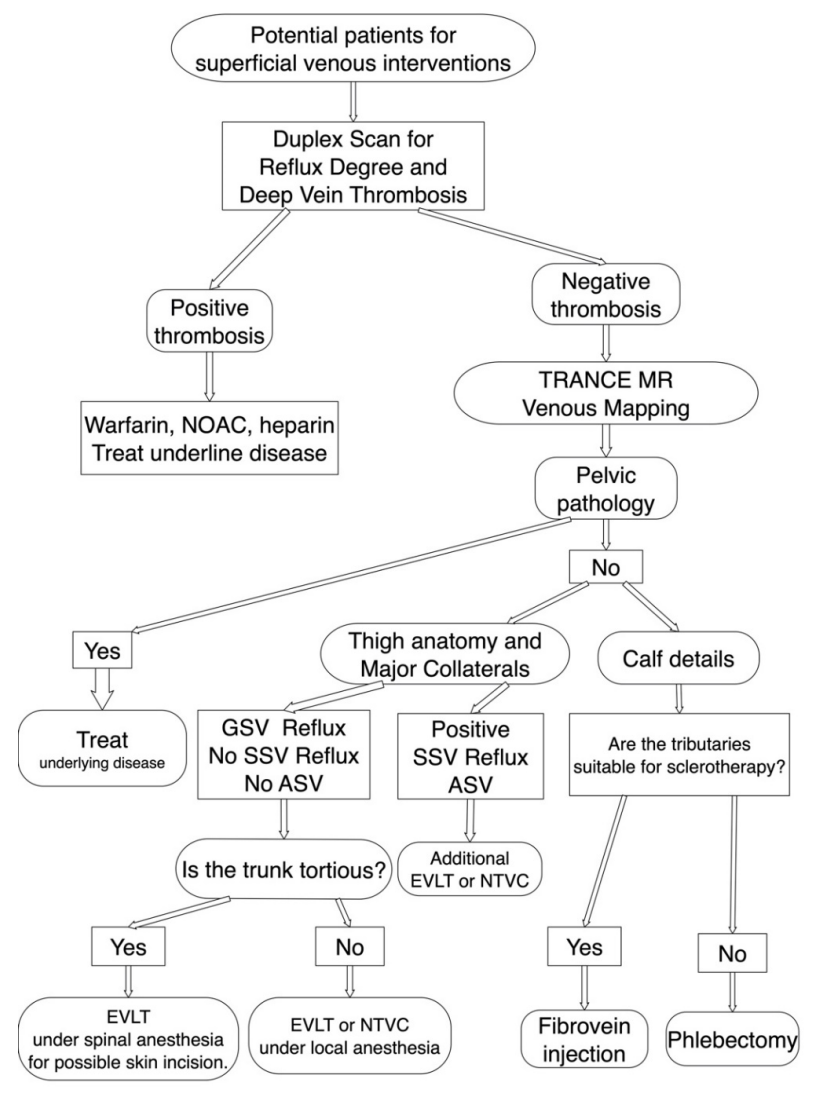

Figure 2. TRANCE MRI venous mapping appliance for preoperative assessment of the superficial venous intervention. Duplex scan was performed first to exclude deep venous thrombosis. If the superficial venous intervention is indicated, we arranged TRANCE MRI mapping to exclude pelvic pathology. Meanwhile, we assess the truncal anatomy, accessory saphenous veins and calf tributaries though TRANCE MRI for complete surgical planning. ASV, accessory saphenous vein; EVLT, Endovenous Laser Treatment; GSV, great saphenous vein; NOAC, non-coumadin oral anticoagulant; NTVC, non-thermal venous closure; SSV, short saphenous vein.

In this study, we included 10 healthy controls and compared the QFflow analysis findings of the patients who received TRANCE-MRI-guided interventions in their left 
leg with the left legs of the controls. SV was higher in the EIV $(p=0.021)$ in the left-legintervention group. The MF was higher in the EIV $(p=0.012)$ and tended to increase in the GSV $(p=0.087)$ in the left-leg-intervention group. SD was longer in the EIV segments $(p=0.019)$. The QFlow of 10 patients who received interventions in their right leg was analyzed, and SV $(p=0.002)$, FFV $(p=0.001)$, and MF $(p=0.001)$ in the GSV were higher in the right-leg-intervention group. We suppose that the patients with venous reflux who were willing to receive intervention had higher SV and MF in the right legs. Moreover, the left-leg-intervention group exhibited higher left EIV flow, implying that the association of pelvic flow, such as pelvic congestions, may be considered in the left legs.

\section{Study Limitations}

The major limitations of this study are its nonrandomized design and small sample size. This TRANCE MRI-guided superficial venous intervention is a new protocol, thus its impact on the clinical outcome is not available yet. However, this is the first series to discuss the use of TRANCE-MRI in conjunction with superficial venous intervention of the legs. In addition to prove the morphological advantage and safety of TRANCE-MRI, this study analyzed QFlow data in surgical scenarios.

\section{Conclusions}

TRANCE-MRI is useful for excluding pelvic lesions, understanding the truncal anatomy, and localizing the major tributaries in the lower extremities. The QFlow data shows that the MF in the GSVs tended to increase in the patients scheduled for surgical intervention compared with the healthy controls. The reversed GSV/PV ratio in the MF could be observed in the QFlow of the morbid limbs. This promising tool may improve the strategy of superficial venous interventions in the lower extremities.

\section{Patents}

This project is under the reviewing process in the Taiwan Intellectual property Office. (No 109126307).

Supplementary Materials: The following are available online at https://www.mdpi.com/article/10 .3390/jpm11080751/s1, Video S1: Both leg for surperficial vein planning.

Author Contributions: Conception and design: Y.-K.H., C.-W.C.; Analysis and interpretation: Y.K.H., C.-W.C., M.Y.W.; Data collection: C.-W.C., Y.-H.T., Y.-F.F., Y.-H.L.; Writing the article: Y.-K.H., C.-W.C.; Statistical analysis: C.-W.C., M.Y.W. and Y.-F.F.; Overall responsibility: Y.-K.H. and C.-W.C. All authors have read and agreed to the published version of the manuscript.

Funding: This study was supported by Chang Gung Memorial Hospital (Contract Nos CORPG6K0131 and CMRPG6K0341).

Institutional Review Board Statement: The Institutional Review Board (IRB) of Chang Gung Memorial Hospital approved this study (IRB number: 201802137B0, 202001213B0 and 202100938B0).

Informed Consent Statement: Informed consent was obtained from all subjects involved in the study.

Data Availability Statement: The data presented in this study are available on request from the corresponding author. The data are not publicly available due to ethical restrictions.

Acknowledgments: We acknowledge Wallace Academiccor.

Conflicts of Interest: The authors declare no conflict of interest. 


$\begin{array}{ll}\text { Abbreviations } & \\ \text { 3D } & \text { three-dimensional } \\ \text { CT } & \text { computed tomography } \\ \text { CTA } & \text { computed tomography angiography } \\ \text { DVT } & \text { deep venous thrombosis } \\ \text { EIV } & \text { external iliac vein } \\ \text { FFV } & \text { forward flow volume } \\ \text { FOV } & \text { field of view } \\ \text { FV } & \text { femoral vein } \\ \text { GSV } & \text { great saphenous vein } \\ \text { IR } & \text { inversion recovery } \\ \text { IRB } & \text { institutional review board } \\ \text { MF } & \text { mean flux } \\ \text { MRI } & \text { magnetic resonance imaging } \\ \text { MRV } & \text { magnetic resonance venography } \\ \text { MV } & \text { mean velocity } \\ \text { NSF } & \text { nephrogenic systemic fibrosis } \\ \text { PV } & \text { popliteal vein } \\ \text { SD } & \text { stroke distance } \\ \text { STIR } & \text { short tau inversion recovery } \\ \text { SV } & \text { stroke volume } \\ \text { TE } & \text { echo time } \\ \text { TOF } & \text { time-of-flight } \\ \text { TR } & \text { repetition time } \\ \text { TRANCE-MRI } & \text { triggered angiography non-contrast-enhanced MRI } \\ \text { TSE } & \text { turbo spin-echo }\end{array}$

\section{References}

1. Tassiopoulos, A.K.; Golts, E.; Oh, D.S.; Labropoulos, N. Current concepts in chronic venous ulceration. Eur. J. Vasc. Endovasc. Surg. 2000, 20, 227-232. [CrossRef] [PubMed]

2. Schleimer, K.; Barbati, M.E.; Grommes, J.; Hoeft, K.; Toonder, I.M.; Wittens, C.H.A.; Jalaie, H. Update on diagnosis and treatment strategies in patients with post-thrombotic syndrome due to chronic venous obstruction and role of endovenous recanalization. J. Vasc. Surg. Venous Lymphat. Disord. 2019, 7, 592-600. [CrossRef]

3. Millan, S.B.; Gan, R.; Townsend, P.E. Venous Ulcers: Diagnosis and Treatment. Am. Fam. Physician 2019, 100, $298-305$.

4. Lin, B.S.; Chen, C.W.; Zhou, S.K.; Tseng, Y.H.; Wang, S.C.; Huang, Y.K. Evaluation of static ulcer on lower extremities using wireless wearable near-infrared spectroscopy device: Effect of deep venous thrombosis on TRiggered Angiography Non-ContrastEnhanced sequence magnetic resonance imaging. Phlebology 2020, 35, 814-823. [CrossRef]

5. Kao, C.C.; Chen, C.W.; Tseng, Y.H.; Tsai, Y.H.; Wang, S.C.; Huang, Y.K. Non-contrast-enhanced magnetic resonance imaging: Objective figures in differentiation between acute and chronic deep venous thrombosis in the lower extremities. Phlebology 2020, 35, 777-783. [CrossRef]

6. $\quad$ Chen, C.W.; Tseng, Y.H.; Lin, C.C.; Kao, C.C.; Wong, M.Y.; Lin, B.S.; Huang, Y.K. Novel Diagnostic Options without Contrast Media or Radiation: Triggered Angiography Non-Contrast-Enhanced Sequence Magnetic Resonance Imaging in Treating Different Leg Venous Diseases. Diagnostics (Basel) 2020, 10, 355. [CrossRef]

7. Asciutto, G.; Mumme, A.; Marpe, B.; Koster, O.; Asciutto, K.C.; Geier, B. MR venography in the detection of pelvic venous congestion. Eur. J. Vasc. Endovasc. Surg. 2008, 36, 491-496. [CrossRef]

8. Lee, Y.L.; Huang, Y.K.; Hsu, L.S.; Chen, P.Y.; Chen, C.W. The use of non-contrast-enhanced MRI to evaluate serial changes in endoleaks after aortic stenting: A case report. BMC Med. Imaging 2019, 19, 82. [CrossRef] [PubMed]

9. Huang, Y.K.; Tseng, Y.H.; Lin, C.H.; Tsai, Y.H.; Hsu, Y.C.; Wang, S.C.; Chen, C.W. Evaluation of venous pathology of the lower extremities with triggered angiography non-contrast-enhanced magnetic resonance imaging. BMC Med. Imaging 2019, 19, 96. [CrossRef]

10. Tseng, Y.H.; Chen, C.W.; Wong, M.Y.; Yang, T.Y.; Lin, B.S.; Ting, H.; Huang, Y.K. Discriminating Reflux from Non-Reflux Diseases of Superficial Veins in Legs by Novel Non-Contrast MR with QFlow Technique. J. Pers. Med. 2021, 11, 242. [CrossRef]

11. Lombardi, P.; Carr, J.C.; Allen, B.D.; Edelman, R.R. Updates in Magnetic Resonance Venous Imaging. Semin. Interv. Radiol. 2021, 38, 202-208. [CrossRef]

12. Gurel, K.; Gurel, S.; Karavas, E.; Buharalioglu, Y.; Daglar, B. Direct contrast-enhanced MR venography in the diagnosis of May-Thurner syndrome. Eur. J. Radiol. 2011, 80, 533-536. [CrossRef]

13. Goodman, L.R. Venous thromboembolic disease: CT evaluation. Q. J. Nucl. Med. 2001, 45, 302-310.

14. Ruehm, S.G.; Zimny, K.; Debatin, J.F. Direct contrast-enhanced 3D MR venography. Eur. Radiol. 2001, 11, 102-112. [CrossRef] 
15. Alfano, G.; Fontana, F.; Ferrari, A.; Solazzo, A.; Perrone, R.; Giaroni, F.; Torricelli, P.; Cappelli, G. Incidence of nephrogenic systemic fibrosis after administration of gadoteric acid in patients on renal replacement treatment. Magn. Reson. Imaging 2020, 70, 1-4. [CrossRef]

16. Schieda, N.; Maralani, P.J.; Hurrell, C.; Tsampalieros, A.K.; Hiremath, S. Updated Clinical Practice Guideline on Use of GadoliniumBased Contrast Agents in Kidney Disease Issued by the Canadian Association of Radiologists. Can. Assoc. Radiol. J. 2019, 70, 226-232. [CrossRef] [PubMed]

17. Ross, M.R.; Pelc, N.J.; Enzmann, D.R. Qualitative phase contrast MRA in the normal and abnormal circle of Willis. AJNR Am. J. Neuroradiol. 1993, 14, 19-25. [PubMed]

18. Giner, J.F.; Sanz-Requena, R.; Florez, N.; Alberich-Bayarri, A.; Garcia-Marti, G.; Ponz, A.; Marti-Bonmati, L. Quantitative phase-contrast MRI study of cerebrospinal fluid flow: A method for identifying patients with normal-pressure hydrocephalus. Neurologia 2014, 29, 68-75. [CrossRef]

19. Gutzeit, A.; Sutter, R.; Froehlich, J.M.; Roos, J.E.; Sautter, T.; Schoch, E.; Giger, B.; Wyss, M.; Graf, N.; von Weymarn, C.; et al. ECG-triggered non-contrast-enhanced MR angiography (TRANCE) versus digital subtraction angiography (DSA) in patients with peripheral arterial occlusive disease of the lower extremities. Eur. Radiol. 2011, 21, 1979-1987. [CrossRef] [PubMed]

20. Suttmeyer, B.; Teichgraber, U.; Rathke, H.; Albrecht, L.; Guttler, F.; Schnackenburg, B.; Hamm, B.; de Bucourt, M. Initial experience with imaging of the lower extremity arteries in an open 1.0 Tesla MRI system using the triggered angiography non-contrast-enhanced sequence (TRANCE) compared to digital subtraction angiography (DSA). Biomed. Tech. (Berl.) 2016, 61, 383-392. [CrossRef]

21. Radlbauer, R.; Salomonowitz, E.; van der Riet, W.; Stadlbauer, A. Triggered non-contrast enhanced MR angiography of peripheral arteries: Optimization of systolic and diastolic time delays for electrocardiographic triggering. Eur. J. Radiol. 2011, 80, 331-335. [CrossRef] [PubMed]

22. Ohno, N.; Miyati, T.; Noda, T.; Alperin, N.; Hamaguchi, T.; Ohno, M.; Matsushita, T.; Mase, M.; Gabata, T.; Kobayashi, S. Fast Phase-Contrast Cine MRI for Assessing Intracranial Hemodynamics and Cerebrospinal Fluid Dynamics. Diagnostics (Basel) 2020, 10, 241. [CrossRef] [PubMed]

23. Altaha, M.A.; Jaskolka, J.D.; Tan, K.; Rick, M.; Schmitt, P.; Menezes, R.J.; Wintersperger, B.J. Non-contrast-enhanced MR angiography in critical limb ischemia: Performance of quiescent-interval single-shot (QISS) and TSE-based subtraction techniques. Eur. Radiol. 2017, 27, 1218-1226. [CrossRef] 